\title{
STRENGTHENED LARGE DEVIATIONS FOR RATIONAL MAPS AND FULL SHIFTS, WITH UNIFIED PROOF
}

\author{
HENRI COMMAN
}

\begin{abstract}
For any hyperbolic rational map and any net of Borel probability measures on the space of Borel probability measures on the Julia set, we show that this net satisfies a strong form of the large deviation principle with a rate function given by the entropy map if and only if the large deviation and the pressure functionals coincide. To each such principles corresponds an expression for the entropy of invariant measures. We give the explicit form of the rate function of the corresponding large deviation principle in the real line for the net of image measures obtained by evaluating the function $\log \left|T^{\prime}\right|$. These results are applied to various examples including those considered in the literature where only upper bounds have been proved. The proof rests on some entropy-approximation property (independent of the net of measures), which in a suitable formulation, is nothing but the hypothesis involving exposed points in Baldi's theorem. In particular, it works verbatim for general dynamical systems. After stating the corresponding general version, as another example we consider the multidimensional full shift for which the above property has been recently proved, and we establish large deviation principles for nets of measures analogous to those of the rational maps case.
\end{abstract}

\section{INTRODUCTION}

The aim of this paper is threefold; it presents large deviation principles arising from two specific dynamical systems, which turn to be typical examples of a general scheme valid for any dynamical system. Let us begin by detailing the first example.

1.1. The case of rational maps. Let $T$ be a hyperbolic rational map of degree at least 2 on the Riemann sphere, and let $J$ be its Julia set. Large deviations for various types of sequences of probability measures on the set $\mathcal{M}(J)$ of Borel probability measures on $J$ have been studied in the literature. We refer here to the distribution of pre-images and periodic points ([16, [15]), and the Birkhoff averages with respect to the measure of maximal entropy ([1]). However, except for this last case, only the large deviation upper-bounds have been proved, and in all cases the rate function has the form

$$
I^{f}(\mu)= \begin{cases}P(T, f)-\mu(f)-h_{\mu}(T) & \text { if } \mu \in \mathcal{M}(J, T) \\ +\infty & \text { if } \mu \in \mathcal{M}(J) \backslash \mathcal{M}(J, T),\end{cases}
$$

where $f$ is some parameter belonging to the set $C(J)$ of real-valued continuous functions on $J$, and $P(T, \cdot), h .(T), \mathcal{M}(J, T)$ denote respectively the pressure map, the entropy map, and the set of invariant elements of $\mathcal{M}(J)$. For each net $\left(\nu_{\alpha}\right)$ of Borel probability measures on $\mathcal{M}(J)$ satisfying a large deviation principle with powers $\left(t_{\alpha}\right)$ and rate function $I^{f}$, the Varadhan's theorem yields the following 
equality,

$$
\forall g \in C(J), \quad \mathbb{L}(\widehat{g}):=\lim t_{\alpha} \log \nu_{\alpha}\left(e^{\widehat{g} / t_{\alpha}}\right)=P(T, f+g)-P(T, f),
$$

where $\widehat{g}(\mu)=\mu(g)$ for all $\mu \in \mathcal{M}(J)$. Our main result establishes the converse, namely, (1.2) implies the large deviation principle with rate function $I^{f}$ (Theorem 3.3). From a well-known result in large deviation theory, the upper-bounds with $I^{f}$ follow from the mere inequality " $\leq$ " in (1.2) with moreover only a upper limit in the L.H.S.; this simple fact allows us to recover the upper bounds proved in [15], [16] ( $c f$. Remark 3.6). The difficult part consists in proving the lower-bounds. The proof rests on the combining of two ingredients. The first is a certain entropy-approximation property for hyperbolic rational maps, namely, any invariant measure can be approximated weakly* and in entropy by a sequence of measures, each one being the unique equilibrium state for some Hölder continuous function; the second one is an application of a general large deviation result in topological vector spaces, the Baldi's theorem. More precisely, the net $\left(\nu_{\alpha}\right)$ is considered as acting on the vector space $\widetilde{\mathcal{M}}(J)$ of signed Borel measures on $J$. The sufficient condition for the large deviation principle in Baldi's theorem involves exposed points and exposing hyperplanes of the functional $\overline{\mathbb{L}}$, defined with a upper limit in (1.2) (cf. 92). Part of this condition is ensured by (1.2), and the remaining is given by the approximation property; indeed, the statement " $\mu$ is an exposed point of the Legendre-Fenchel transform $\overline{\mathbb{L}}^{*}$ with exposing hyperplane $\widehat{g}^{\prime}$ is just an abstract formulation of " $\mu$ is the unique equilibrium state for $f+g$ ".

The main advantage in having the large deviation principle follows from the fact that the rate function $I^{f}$ is affine, which implies that convex open sets containing some invariant measures are $I^{f}$-continuity sets ( $c f$. 92 ), and so we get limits on these sets. Furthermore, in view of the form of the rate function (1.1), the above entropy-approximation property is nothing but a continuity property of $I^{f}$; this gives a strengthened form of the large deviation principle in the sense that the limit on convex open sets is given up to $\varepsilon$ by $I^{f}\left(\mu_{f+g_{\varepsilon}}\right)$, where $\mu_{f+g_{\varepsilon}}$ is the unique equilibrium state for some Hölder continuous function $f+g_{\varepsilon}$. As a consequence, to each of such large deviation principles and for each invariant measure $\mu$, corresponds an expression of the entropy $h_{\mu}(T)$ in terms of these limits.

Practically, to each way of obtaining the pressure in the sense of (1.2) corresponds a large deviation principle. This is the case for every $f \in C(J)$ with the sequence

$$
\nu_{n, f}:=\sum_{y \in J_{n}} p_{n, f}(y) \delta_{\frac{1}{n}\left(\delta_{y}+\ldots+\delta_{T^{n-1} y}\right)},
$$

where $J_{n}$ is a maximal $(\varepsilon, n)$-separated set with $\varepsilon$ small enough, and

$$
p_{n, f}(y)=\frac{e^{f(y)+\ldots+f\left(T^{n-1} y\right)}}{\sum_{z \in J_{n}} e^{f(z)+\ldots+f\left(T^{n-1} z\right)}} .
$$

This case is new and constitutes our first example (4.1). It is generic in the sense that it suffices to replace $J_{n}$ either by the set of $n$-preimages or $n$-periodic points (with moreover $f$ Hölder continuous) in order to get the same results for the corresponding measures (44.2); this improves [15] and [16] by giving the lower-bounds. The large deviation principle concerning the distribution of Birkhoff averages with respect to the measure of maximal entropy (proved in [11]) is a direct consequence of our main result (Remark 4.1). The expressions of the entropy corresponding to the above examples are given by (4.7), (4.8), (4.18). 
Also, by the so-called contraction principle with any $k \in C(J)([4],[3)$, we get a level-1 large deviation principle for the sequence $\left(\widehat{k}\left[\nu_{n}\right]\right)$ of image measures (Corollary [3.4). The case $f=-t \log \left|T^{\prime}\right|$ and $k=\log \left|T^{\prime}\right|$ is studied in detail (Theorem 3.5); in particular, this yields various formulas for $h_{\mu_{s}}(T)$, where $\mu_{s}$ is the equilibrium state for $-s \log \left|T^{\prime}\right|(c f$. (4.14), (4.16), (4.19)). Some of these results are analogues of those of 8 . concerning unimodal interval maps; it is the case for the existence of a strictly negative limit in (4.15) with $t=0$ and (4.20), but we have more here since the bounds are explicit.

We draw attention to the fact that (except those obtained by contraction as above) we are concerned here only with level-2 large deviation principles (i.e. in the space $\mathcal{M}(J)$ ), and we do not take up level-1 large deviations (i.e. in the real line) with some fixed potential and reference measure (see [19, [12] and references therein for recent developments on this topic).

1.2. Generalization and the example of the multidimensional full shift. In the last part of the paper ( $(5)$, we observe that the proof of the main theorem (Theorem 3.3) works as well for any dynamical system $(\Omega, \tau)$ (where $\Omega$ is the phase space and $\tau$ the semi-group or group action) satisfying a similar entropyapproximation property, and for any net of Borel probability measures on $\mathcal{M}(\Omega)$ satisfying (1.2) (after obvious changes of notations). Indeed, roughly speaking, $I^{f}$ is nothing but the Legendre-Fenchel transform of the pressure (Lemma 3.1) seen as the large deviation functional associated to the underlying net of measures, so that the above conditions ensure that the hypotheses of Baldi's theorem are satisfied and the large deviations follow. The corresponding result is stated in Theorem 5.2 as for rational maps, it constitutes a strong form of a large deviation principle with rate function $I^{f}$, since for each invariant measure $\mu, I^{f}(\mu)$ can be approximated by $I^{f}\left(\mu_{i}\right)$ where $\mu_{i}$ is the unique equilibrium state for some potential (Remark 5.3). Again here, only the inequality " $\leq$ " in (1.2) with a upper limit in the L.H.S. is required to get the upper bounds with $I^{f}$.

It turns out that recently Gurevich and Tempelman ([6], Theorem 1) proved the entropy-approximation property for the multidimensional full shift. As a consequence, we obtain large deviation principles for nets constructed with maximal separated sets (resp. periodic configurations), exactly as for rational maps; both results are new (Theorem 5.7).

1.3. Important remark. Although some of the large deviation principles concerning hyperbolic rational maps proved here can be proved as well using Kifer's techniques (9], 10]), this is generally not the case. Indeed, for any dynamical system $(\Omega, \tau)$ (as in $\$ 5$ ) and any $f \in C(\Omega)$, these techniques combined with the general version of Lemma 3.1 yield the following result: If there exists a countably generated dense vector subspace $V \subset C(\Omega)$ such that $f+g$ has a unique equilibrium state for all $g \in V$, then any net of Borel probability measures on $\mathcal{M}(\Omega)$ such that

$$
\forall g \in V, \quad \mathbb{L}(\widehat{g})=P^{\tau}(f+g)-P^{\tau}(f),
$$

satisfies a large deviation principle with rate function $I^{f}$. In the case of hyperbolic rational maps and under the assumption that $f$ is Hölder continuous, it suffices to take $V$ the space of such functions and to apply the above result. However, whatever $V$ satisfying the above conditions, taking $g=0$ implies that $f$ has a unique equilibrium state, and so this method does not work for general $f$, as in 4.1 . This observation is valid a fortiori for the multidimensional time setting, as in $\sqrt[4.1]{5 \text { where }}$ 
Theorem 5.7 works for every $f \in C(\Omega)$. So, the entropy-approximation property, a purely dynamical one (i.e. independent of the measures) gives a direct way to get large deviations for any net of measures satisfying (1.3), which furthermore can work when usual techniques fail. It occurs in the two systems of distinct nature considered here, and a natural question arises about the generality of such a property; also, it would be interesting to study the relation with the above mentioned Kifer's condition.

1.4. Organization. In 42 we recall basic facts of large deviation theory, and in particular Baldi's theorem. The next two sections deal with rational maps; 83 presents the general results, and $\$ 4$ the examples. The last section treats the generalization and the example of multidimensional full shift.

\section{Preliminaries}

Let $X$ be a Hausdorff regular topological space, let $\left(\nu_{\alpha}\right)$ be a net of Borel probability measures on $X$, and let $\left(t_{\alpha}\right)$ be a net in $] 0,+\infty$ [ converging to 0 . We say that $\left(\nu_{\alpha}\right)$ satisfies a large deviation principle with powers $\left(t_{\alpha}\right)$ if there exists a $[0,+\infty]$ valued lower semi-continuous function $I$ on $X$ such that

$$
\varlimsup t_{\alpha} \log \nu_{\alpha}(F) \leq-\inf _{x \in F} I(x) \leq-\inf _{x \in G} I(x) \leq \underline{\lim } t_{\alpha} \log \nu_{\alpha}(G)
$$

for all closed sets $F \subset X$ and all open sets $G \subset X$ with $F \subset G$; such a function $I$ is then unique (called the rate function) and given for each $x \in X$ by

$$
-I(x)=\inf _{G \in \mathcal{G}_{x}} \underline{\lim } t_{\alpha} \log \nu_{\alpha}(G)=\inf _{G \in \mathcal{G}_{x}} \varlimsup t_{\alpha} \log \nu_{\alpha}(G),
$$

where $\mathcal{G}_{x}$ is any local basis at $x$. When the above large deviation principle holds, we have

$$
\lim t_{\alpha} \log \nu_{\alpha}(Y)=-\inf _{x \in Y} I(x)
$$

for all Borel sets $Y \subset X$ satisfying $\inf _{x \in \operatorname{Int} Y} I(x)=\inf _{x \in \bar{Y}} I(x)$ (where Int $Y$ denotes the interior of $Y$ ), and we can replace $Y$ by Int $Y$ (resp. $\bar{Y}$ ) in (2.2) (such sets $Y$ are called $I$-continuity sets).

The large deviation functional $\overline{\mathbb{L}}$ associated to $\left(\nu_{\alpha}\right)$ and $\left(t_{\alpha}\right)$ is defined on the set of $[-\infty,+\infty[$-valued Borel functions $h$ on $X$ by

$$
\overline{\mathbb{L}}(h)=\varlimsup t_{\alpha} \log \nu_{\alpha}\left(e^{h / t_{\alpha}}\right) .
$$

Note that $\overline{\mathbb{L}}$ is continuous with respect to the uniform metric. We write $\mathbb{L}(h)$ when the limit exists. Assume furthermore that $X$ is a real topological vector space with topological dual $X^{*}$, and let $\overline{\mathbb{L}}^{*}$ denotes the Legendre-Fenchel transform of $\overline{\mathbb{L}}_{\mid X^{*}}$ (this restriction is the so-called "generalized log-moment generating function"). An element $x \in X$ is an exposed point of $\overline{\mathbb{L}}^{*}$ if there exists $\lambda \in X^{*}$ (called exposing hyperplane) such that

$$
\forall y \neq x, \quad \lambda(x)-\overline{\mathbb{L}}^{*}(x)>\lambda(y)-\overline{\mathbb{L}}^{*}(y) .
$$

The main tool to derive the large deviation lower bounds is the following classical result of Baldi (1], 4]; see also [2] for a strengthened version). We shall apply it with $X=\widetilde{\mathcal{M}}(\Omega)$ (where $\Omega$ is the phase space of the system) by showing that the approximation property mentioned in $\$ 1$ implies the condition on exposed points. We recall that $\left(\nu_{\alpha}\right)$ is said to be exponentially tight with respect to $\left(t_{\alpha}\right)$ if for each real $M$ there exists a compact $K_{M} \subset X$ such that $\varlimsup t_{\alpha} \log \nu_{\alpha}\left(X \backslash K_{M}\right)<M$; 
our nets of measures are trivially exponentially tight since they are constituted by measures supported by the compact set $\mathcal{M}(\Omega)$ (in particular, the boundness condition on $\overline{\mathbb{L}}_{\mid X^{*}}$ is always satisfied).

Theorem 2.1. (Baldi) Let $X$ be a real Hausdorff topological vector space and assume that $\left(\nu_{\alpha}\right)$ is exponentially tight with respect to $\left(t_{\alpha}\right)$. Let $\mathcal{E}$ be the set of exposed points $x$ of $\overline{\mathbb{L}}^{*}$ for which there is an exposing hyperplane $\lambda_{x}$ such that $\mathbb{L}\left(\lambda_{x}\right)$ exists and $\overline{\mathbb{L}}\left(c \lambda_{x}\right)<+\infty$ for some $c>1$. If $\inf _{G} \overline{\mathbb{L}}^{*}=\inf _{G \cap \mathcal{E}} \overline{\mathbb{L}}^{*}$ for all open sets $G \subset X$, then $\left(\nu_{\alpha}\right)$ satisfies a large deviation principle with powers $\left(t_{\alpha}\right)$ and rate function $\bar{L}^{*}$.

\section{Hyperbolic RATIONAL MAPS AND BALDI'S THEOREM}

Throughout this section and the next one, $T$ is a hyperbolic rational map of degree $d \geq 2$ (i.e. expanding on its Julia set $J$ ), and $C(J)$ the set of real-valued continuous functions on $J$ provided with the uniform topology. We denote by $\widetilde{\mathcal{M}}(J)$ the vector space of signed Borel measures on $J$ provided with the weak*topology, and by $\mathcal{M}(J)$ (resp. $\mathcal{M}(J, T)$ ) the set of Borel probability measures on $J$ (resp. T-invariant elements of $\mathcal{M}(J)$ ) endowed with the induced topology. For any $g \in C(J), P(T, g), \mathcal{M}_{g}(J, T)$ and $h_{\mu}(T)$ stand for the topological pressure of $g$, the set of equilibrium states for $g$, and the measure-theoretic entropy of $T$ with respect to $\mu$, respectively; we recall that $\mathcal{M}_{g}(J, T)$ has a unique element when $g$ is Hölder continuous. We denote by $\widehat{g}$ the map defined on $\widetilde{\mathcal{M}}(J)$ by $\widehat{g}(\mu)=\mu(g)$. For each $f \in C(J)$ we define a map $Q_{f}$ on $C(J)$ by

$$
Q_{f}(g)=P(T, f+g)-P(T, f),
$$

and note that $Q_{f}$ is real-valued, convex, and continuous with respect to the uniform metric. The Legendre-Fenchel transform $Q_{f}{ }^{*}$ of $Q_{f}$ will appear in the sequel as the rate function of our level-2 large deviation principles; note that $Q_{f}{ }^{*}$ vanishes exactly on $\mathcal{M}_{f}(J, T)$, as shows the following lemma.

Lemma 3.1. We have

$$
Q_{f}^{*}(\mu)= \begin{cases}P(T, f)-\mu(f)-h_{\mu}(T) & \text { if } \mu \in \mathcal{M}(J, T) \\ +\infty & \text { if } \mu \in \widetilde{\mathcal{M}}(J) \backslash \mathcal{M}(J, T) .\end{cases}
$$

For each pair of functions $f, g$ in $C(J)$ and each $\mu \in \mathcal{M}(J), \mu$ is an equilibrium state for $f+g$ if and only if $Q_{f}(g)=\mu(g)-Q_{f}{ }^{*}(\mu)$.

Proof. Put

$$
U(\mu)= \begin{cases}-\mu(f)-h_{\mu}(T) & \text { if } \mu \in \mathcal{M}(J, T) \\ +\infty & \text { if } \mu \in \widetilde{\mathcal{M}}(J) \backslash \mathcal{M}(J, T) .\end{cases}
$$

We have

$$
P(T, f+g)=\sup _{\mu \in \mathcal{M}(J, T)}\left\{\mu(f+g)+h_{\mu}(T)\right\}=\sup _{\mu \in \widetilde{\mathcal{M}}(J)}\{\mu(g)-U(\mu)\}
$$


and since the entropy map is affine upper semi-continuous, $U$ is convex lower semicontinuous and $]-\infty,+\infty]$-valued. By the duality theorem, $U$ is the LegendreFenchel transform of the map $g \rightarrow P(T, f+g)$, that is for each $\mu \in \widetilde{\mathcal{M}}(J)$,

$$
\begin{aligned}
U(\mu) & =\sup _{g \in C(J)}\{\mu(g)-P(T, f+g)\}=\sup _{g \in C(J)}\left\{\mu(g)-P(T, f)-Q_{f}(g)\right\} \\
& =-P(T, f)+\sup _{g \in C(J)}\left\{\mu(g)-Q_{f}(g)\right\}=-P(T, f)+Q_{f}{ }^{*}(\mu),
\end{aligned}
$$

which proves the first assertion. The last assertion follows from the equalities

$$
\begin{aligned}
P(T, f+g)-h_{\mu}(T)-\mu(f+g) & =Q_{f}(g)+P(T, f)-h_{\mu}(T)-\mu(f+g) \\
& =Q_{f}(g)+Q_{f}{ }^{*}(\mu)-\mu(g) .
\end{aligned}
$$

The following approximation property is proved in [11, Theorem 8].

Theorem 3.2. (Lopes) For each $\mu \in \mathcal{M}(J, T)$ there exists a sequence $\left(k_{i}\right)$ of Hölder continuous functions on $J$ such that the sequence $\left(\mu_{i}\right)$ of their respective equilibrium states satisfies $\lim \mu_{i}=\mu$ and $\lim h_{\mu_{i}}(T)=h_{\mu}(T)$.

The main result of this section is the following theorem, where the large deviation principle is obtained by a direct application of Baldi's theorem, once observed that the condition on exposed points follows from Theorem 3.2

Theorem 3.3. Let $f \in C(J)$, let $\left(\nu_{\alpha}\right)$ be a net of Borel probability measures on $\mathcal{M}(J)$, let $\left(t_{\alpha}\right)$ be a net in $] 0,+\infty[$ converging to 0 , and assume that

$$
\lim t_{\alpha} \log \nu_{\alpha}\left(e^{\widehat{g} / t_{\alpha}}\right)=Q_{f}(g)
$$

for all $g$ in a dense subset of $C(J)$.

a) The net $\left(\nu_{\alpha}\right)$ satisfies a large deviation principle with power $\left(t_{\alpha}\right)$ and rate function

$$
I^{f}(\mu)= \begin{cases}P(T, f)-\mu(f)-h_{\mu}(T) & \text { if } \mu \in \mathcal{M}(J, T) \\ +\infty & \text { if } \mu \in \mathcal{M}(J) \backslash \mathcal{M}(J, T) .\end{cases}
$$

Moreover, for each convex open set $G \subset \mathcal{M}(J)$ containing some invariant measure we have

$$
\lim t_{\alpha} \log \nu_{\alpha}(G)=\lim t_{\alpha} \log \nu_{\alpha}(\bar{G})=-\inf _{\mu \in \bar{G}} I^{f}(\mu)=-\inf _{\mu \in G \cap \mathcal{E}^{\prime}} I^{f}(\mu),
$$

where $\mathcal{E}^{\prime}$ is the set of equilibrium states of all Hölder continuous functions on $J$. In particular, for each $\mu \in \mathcal{M}(J, T)$ and each convex local basis $\mathcal{G}_{\mu}$ at $\mu$ we obtain

$$
h_{\mu}(T)=P(T, f)-\mu(f)+\inf \left\{\lim t_{\alpha} \log \nu_{\alpha}(G): G \in \mathcal{G}_{\mu}\right\} .
$$

b) Each limit point of $\left(\nu_{\alpha}\right)$ has its support included in $\mathcal{M}_{f}(J, T)$; in particular, $\lim \nu_{\alpha}=\delta_{\mu_{f}}$ when $f$ has a unique equilibrium sate $\mu_{f}$.

Proof. a) We consider $\left(\nu_{\alpha}\right)$ as a net of Borel probability measures on $\widetilde{\mathcal{M}}(J)$, and note that the corresponding large deviation functional (with slight abuse of notation we denoted also by $\overline{\mathbb{L}}$ ) satisfies $\overline{\mathbb{L}}(\cdot)=\overline{\mathbb{L}}\left(\odot_{\mid \mathcal{M}(J)}\right)$. Since $\sup _{J}|g|=\sup _{\mathcal{M}(J)}|\widehat{g}|$, the maps $g \mapsto \overline{\mathbb{L}}(\widehat{g})-Q_{f}(g)$ and $g \mapsto \underline{\mathbb{L}}(\widehat{g})-Q_{f}(g)$ are continuous (where $\underline{\mathbb{L}}$ is defined 
replacing the upper limit by a lower limit in (2.3)), and so the general hypothesis implies the existence of $\mathbb{L}(\widehat{g})$ with the equality $\mathbb{L}(\widehat{g})=Q_{f}(g)$ for all $g \in C(J)$. Consequently, we have

$$
\mathbb{L}^{*}=Q_{f}^{*},
$$

and the large deviation upper-bounds in $\widetilde{\mathcal{M}}(J)$ with the function $\mathbb{L}^{*}$ follow from a well-known result in topological vector spaces, namely, Theorem 4.5.3 of [4] (cf. Remark 3.6). Let $\mathcal{E}$ denote the set of exposed points of $\mathbb{L}^{*}$, and note that $\mathcal{E}$ coincides with the set denoted by the same symbol in Theorem 2.1 since $\mathbb{L}_{\mid \widetilde{\mathcal{M}}(J)^{*}}$ is here realvalued. For every $\mu \in \mathcal{M}(J)$, (3.2) and the last assertion of Lemma 3.1 show that $\mu$ is the unique equilibrium state for $f+g$ if and only if $\mu \in \mathcal{E}$ with exposing hyperplane $\widehat{g}$. Let $\mathcal{E}^{\prime}$ denote the subset of $\mathcal{E}$ constituted by the equilibrium states of all Hölder continuous functions. Putting $k_{i}=f+g_{i}$ in Theorem 3.2, it follows from the expression of $Q_{f}{ }^{*}$ given by Lemma 3.1 that for each $\mu \in \mathcal{M}(J, T)$, there exists a sequence $\left(f+g_{i}\right)$ in $C(J)$ and a sequence $\left(\mu_{i}\right)$ in $\mathcal{M}(J, T)$ such that

(i) $\mu_{i} \in \mathcal{E}^{\prime}$ (with exposing hyperplane $\widehat{g}_{i}$, or equivalently $\mu_{i}$ is the unique equilibrium state for $\left.f+g_{i}\right)$;

(ii) $\lim \mu_{i}=\mu$;

(iii) $\lim \mathbb{L}^{*}\left(\mu_{i}\right)=\mathbb{L}^{*}(\mu)$.

Since $\mathcal{E}^{\prime} \subset \mathcal{E} \subset \mathcal{M}(J, T)$ and $\mathbb{L}^{*}$ is infinite-valued outside $\mathcal{M}(J, T)$ we have for each open set $G \subset \mathcal{M}(J)$,

$$
\inf _{G} \mathbb{L}^{*}=\inf _{G \cap \mathcal{M}(J, T)} \mathbb{L}^{*} \leq \inf _{G \cap \mathcal{E}} \mathbb{L}^{*} \leq \inf _{G \cap \mathcal{E}^{\prime}} \mathbb{L}^{*},
$$

and the properties (i)-(iii) show that both above inequalities are equalities. Consequently, all the hypotheses of Theorem 2.1 are fulfilled, and so $\left(\nu_{\alpha}\right)$ satisfies a large deviation principle in $\widetilde{\mathcal{M}}(J)$ with powers $\left(t_{\alpha}\right)$ and rate function $\mathbb{L}^{*}$. Since $\mathcal{M}(J)$ is closed in $\widetilde{\mathcal{M}}(J)$, the large deviation principle holds in $\mathcal{M}(J)$ with rate function $\mathbb{L}^{*} \mid \mathcal{M}(J)$ (4], Lemma 4.1.5); this proves the first assertion since $I^{f}=\mathbb{L}^{*} \mid \mathcal{M}(J)$ by (3.2) and Lemma 3.1. Let $G \subset \mathcal{M}(J)$ be a convex open set containing some invariant measure $\mu^{\prime}$. Let $\mu \in \bar{G}$ satisfying $I^{f}(\mu)=\inf _{\bar{G}} I^{f}$, and suppose that $I^{f}(\mu)<\inf _{G} I^{f}$; in particular $\mu \in \mathcal{M}(J, T) \cap \bar{G} \backslash G$. Let $\lambda_{n}$ be a sequence in ]0,1[ converging to 0 , put $\mu_{n}=\lambda_{n} \mu^{\prime}+\left(1-\lambda_{n}\right) \mu$, and note that $\mu_{n} \in G$ (24], pp. 38). We have $\lim \mu_{n}=\mu$ and $\lim I^{f}\left(\mu_{n}\right)=I^{f}(\mu)$ since $I^{f}$ is affine and real-valued on $\mathcal{M}(J, T)$, which gives the contradiction; therefore $\inf _{G} I^{f}=\inf _{\bar{G}} I^{f}$, and since (3.3) holds with equalities we get

$$
\inf _{\bar{G}} I^{f}=\inf _{G \cap \mathcal{E}^{\prime}} I^{f}
$$

The two last assertions are direct consequences of the above equality and the large deviation principle $(c f$. (2.2), (2.1)).

b) Let $\left(\nu_{\beta}\right)$ be a subnet of $\left(\nu_{\alpha}\right)$ converging to some $\nu$, let $\mu \in \mathcal{M}(J, T) \backslash \mathcal{M}_{f}(J, T)$, and let $G$ be an open set satisfying

$$
\mu \in G \subset \bar{G} \subset \mathcal{M}(J, T) \backslash \mathcal{M}_{f}(J, T) .
$$

The large deviation upper bounds yields

$$
\varlimsup t_{\alpha} \log \nu_{\alpha}(G) \leq \varlimsup t_{\alpha} \log \nu_{\alpha}(\bar{G})<0,
$$

hence

$$
\lim \nu_{\beta}(G)=\nu(G)=0,
$$

which shows that $\operatorname{supp}(\nu) \subset \mathcal{M}_{f}(J, T)$. 
Corollary 3.4. Under the hypotheses of Theorem 3.3, for each $k \in C(J)$ the net of image measures $\left(\widehat{k}\left[\nu_{\alpha}\right]\right)$ satisfies a large deviation principle in $\mathbb{R}$ with powers $\left(t_{\alpha}\right)$ and convex rate function

$$
\forall x \in \mathbb{R}, \quad I_{k}^{f}(x)=\inf _{\{\mu \in \mathcal{M}(J): \mu(k)=x\}} I^{f}(\mu) .
$$

Explicitly, we have

$$
\varlimsup t_{\alpha} \log \nu_{\alpha}\{\mu \in \mathcal{M}(J): \mu(k) \in C\} \leq-\inf _{x \in C} I_{k}^{f}(x)
$$

for all closed sets $C \subset \mathbb{R}$, and

$$
\underline{\lim } t_{\alpha} \log \nu_{\alpha}\{\mu \in \mathcal{M}(J): \mu(k) \in U\} \geq-\inf _{x \in U} I_{k}^{f}(x)
$$

for all open sets $U \subset \mathbb{R}$. Moreover, for each $\mu \in \mathcal{M}(J, T)$ and each $\varepsilon>0$ small enough, we have

$$
\lim t_{\alpha} \log \nu_{\alpha}\left(G_{k, \mu, \varepsilon}\right)=\lim t_{\alpha} \log \nu_{\alpha}\left(\overline{G_{k, \mu, \varepsilon}}\right)=-\inf _{G_{k, \mu, \varepsilon}} I^{f}=-\inf _{G_{k, \mu, \varepsilon}} I^{f},
$$

where $G_{k, \mu, \varepsilon}=\left\{\mu^{\prime} \in \mathcal{M}(J):\left|\mu^{\prime}(k)-\mu(k)\right|>\varepsilon\right\}$.

Proof. The large deviation principle with rate function $I_{k}^{f}$ follows from the contraction principle (4], Theorem 4.2.1) applied to $\left(\nu_{\alpha}\right)$ with $\widehat{k}$. For each pair of reals $x_{1}, x_{2}$ and each $\left.\beta \in\right] 0,1[$ we have

$$
\begin{gathered}
I_{k}^{f}\left(\beta x_{1}+(1-\beta) x_{2}\right)=\inf \left\{I^{f}(\mu): \mu \in \mathcal{M}(J, T), \mu(k)=\beta x_{1}+(1-\beta) x_{2}\right\} \\
\leq \inf \left\{I^{f}\left(\beta \mu_{1}+(1-\beta) \mu_{2}\right): \mu_{1} \in \mathcal{M}(J, T), \mu_{2} \in \mathcal{M}(J, T), \mu_{1}(k)=x_{1}, \mu_{2}(k)=x_{2}\right\} \\
=\inf \left\{\beta I^{f}\left(\mu_{1}\right)+(1-\beta) I^{f}\left(\mu_{2}\right): \mu_{1} \in \mathcal{M}(J, T), \mu_{2} \in \mathcal{M}(J, T), \mu_{1}(k)=x_{1}, \mu_{2}(k)=x_{2}\right\} \\
\leq \beta I_{k}^{f}\left(x_{1}\right)+(1-\beta) I_{k}^{f}\left(x_{2}\right)
\end{gathered}
$$

and hence $I_{k}^{f}$ is convex. For each $\mu \in \mathcal{M}(J, T)$ and each $\delta>0$ we put

$$
G_{1, \delta}=\left\{\mu^{\prime} \in \mathcal{M}(J): \mu^{\prime}(k)-\mu(k)>\delta\right\}
$$

and

and note that

$$
G_{2, \delta}=\left\{\mu^{\prime} \in \mathcal{M}(J): \mu^{\prime}(k)-\mu(k)<-\delta\right\}
$$

$$
\forall \delta^{\prime}<\delta, \quad G_{j, \delta^{\prime}} \supset \overline{G_{j, \delta}}, \quad(j \in\{1,2\}) .
$$

First assume that $\left(G_{1, \delta} \cup G_{2, \delta}\right) \cap \mathcal{M}(J, T) \neq \emptyset$ for some $\delta>0$. If $G_{1, \delta} \cap G_{2, \delta}$ contains some invariant measure then (3.4) with $\varepsilon=\delta$ follows from (3.1) applied to $G_{1, \delta} \cup G_{2, \delta}$. Clearly the same holds for all $\left.\left.\varepsilon \in\right] 0, \delta\right]$ by (3.5). If $G_{j, \delta} \cap \mathcal{M}(J, T)=\emptyset$ for some $j \in\{1,2\}$ (say $j=1$ ), then either $G_{1, \delta^{\prime}} \cap \mathcal{M}(J, T) \neq \emptyset$ for all $\delta^{\prime}<\delta$, and we fall in the preceding case with $\delta^{\prime}$ in place of $\delta$; either $\overline{G_{1, \delta}} \cap \mathcal{M}(J, T)=\emptyset$ and the large deviation upper bounds yields $\overline{\lim } t_{\alpha} \log \nu_{\alpha}\left(\overline{G_{1, \delta}}\right)=-\infty$, and (3.4) follows from (3.1) applied to $G_{2, \delta}$. When $\left(G_{1, \delta} \cup G_{2, \delta}\right) \cap \mathcal{M}(J, T)=\emptyset$ for all $\delta>0$, from (3.5) we obtain

$$
\forall \delta>0, \quad \overline{G_{1, \delta} \cup G_{2, \delta}} \cap \mathcal{M}(J, T)=\emptyset,
$$

and (3.4) follows from the upper bounds applied to $\overline{G_{1, \delta} \cup G_{2, \delta}}$ for all $\delta>0$ (note that in this case, (3.4) takes an infinite value, and $\widehat{k}_{\mid \mathcal{M}(J, T)}$ is the constant function equals to $\mu(k))$. 
For each real $t$, we put $k_{t}=-t \log \left|T^{\prime}\right|$ (i.e. $\widehat{k_{-1}} \mid \mathcal{M}(J, T)=\chi$ is the Lyapunov map), and we write $P(t)$ for $P\left(T, k_{t}\right)$. The map $t \mapsto P(t)$ is real analytic, strictly decreasing, and strictly convex when $T$ is not conjugate to $z \mapsto z^{ \pm d}$ (in this last case $P(t)=\log d(1-t))$. The map $k_{t}$ has a unique equilibrium state, which we denote in what follows simply by $\mu_{t}$, in place of $\mu_{k_{t}}$ ([21], [7]). We put $\chi_{\text {inf }}=$ $\inf \{\chi(\mu): \mu \in \mathcal{M}(J, T)\}, \chi_{\text {sup }}=\sup \{\chi(\mu): \mu \in \mathcal{M}(J, T)\}$, and recall that $\chi_{\text {inf }}=$ $\inf \left\{-P^{\prime}(t): t \in \mathbb{R}\right\}>0$ and $\chi_{\text {sup }}=\sup \left\{-P^{\prime}(t): t \in \mathbb{R}\right\}$. In the following, we specify Corollary 3.4 by taking $f=k_{t}$ and $k=k_{-1}$.

Theorem 3.5. Assume that the hypotheses of Theorem 3.3 hold with $f=k_{t}$ for some real $t$. Then the net $\left(\widehat{k_{-1}}\left[\nu_{\alpha}\right]\right)$ satisfies a large deviation principle with rate function

$$
I_{k_{-1}}^{k_{t}}(x)= \begin{cases}0 & \text { if } x=\log d \\ +\infty & \text { if } x \neq \log d\end{cases}
$$

when $T$ is conjugate to $z \mapsto z^{ \pm d}$, and

$$
I_{k_{-1}}^{k_{t}}(x)= \begin{cases}P(t)+t x-h_{\mu_{s_{x}}}(T) & \text { if } \chi_{\mathrm{inf}}<x<\chi_{\mathrm{sup}} \\ +\infty & \text { otherwise }\end{cases}
$$

in all others cases, where $s_{x}$ is the unique real such that $\chi\left(\mu_{s_{x}}\right)=x ; I_{k_{-1}}^{k_{t}}$ is then strictly convex on $] \chi_{\mathrm{inf}}, \chi_{\mathrm{sup}}[$, and essentially smooth. Moreover, we have

$$
\lim \nu_{\alpha}\left(\widehat{k_{-1}}\right)=\chi\left(\mu_{t}\right) .
$$

Proof. For each real $s$ let $u_{s}$ be the map defined on $\mathbb{R}$ by $u_{s}(x)=s x$. We have

$$
\begin{gathered}
L(s):=\lim t_{\alpha} \log \widehat{k_{-1}}\left[\nu_{\alpha}\right]\left(e^{u_{s} / t_{\alpha}}\right)=\lim t_{\alpha} \log \nu_{\alpha}\left(e^{u_{s} \circ \widehat{k_{-1}} / t_{\alpha}}\right)=\mathbb{L}\left(u_{s} \circ \widehat{k_{-1}}\right) \\
=\sup _{\mu \in \mathcal{M}(J)}\left\{u_{s} \circ \widehat{k_{-1}}(\mu)-I^{f}(\mu)\right\}=\sup _{\mu \in \mathcal{M}(J, T)}\left\{\mu\left(s k_{-1}\right)-P\left(T, k_{t}\right)+\mu\left(k_{t}\right)+h_{\mu}(T)\right\} \\
=P(t-s)-P(t),
\end{gathered}
$$

where the existence of the limits and the fourth equality follow from the large deviation principle for $\left(\nu_{\alpha}\right)$ and the Varadhan's theorem applied to the bounded continuous function $u_{s} \circ \widehat{k_{-1} \mid \mathcal{M}(J)}(c f$. [4], [3]). The map $L$ is then differentiable on $\mathbb{R}$, and consequently $I_{k_{-1}}^{k_{t}}=L^{*}$ by Gärtner-Ellis theorem ([4]). This proves (3.6) since $P(t)=\log d(1-t)$ when $T$ is conjugate to $z \mapsto z^{ \pm d}$. Assume that $T$ is not conjugate to $z \mapsto z^{ \pm d}$. We have

$$
\forall \mu \in \mathcal{M}(J, T), \quad I^{k_{t}}(\mu)=P(t)+t \chi(\mu)-h_{\mu}(T)
$$

and

$$
\begin{gathered}
P\left(s_{\chi(\mu)}\right)=-s_{\chi(\mu)} \chi\left(\mu_{s_{\chi(\mu)}}\right)+h_{\mu_{s_{\chi}(\mu)}}(T)=-s_{\chi(\mu)} \chi(\mu)+h_{\mu_{s_{\chi}(\mu)}}(T) \\
\geq-s_{\chi(\mu)} \chi(\mu)+h_{\mu}(T),
\end{gathered}
$$

hence

$$
\forall \mu \in \mathcal{M}(J, T), \quad h_{\mu}(T) \leq h_{\mu_{s_{\chi}(\mu)}}(T) .
$$

Combining (3.8) and (3.9) we get

$$
\forall x \in \mathbb{R}, \quad I_{k_{-1}}^{k_{t}}(x)=\inf _{\mu \in \mathcal{M}(J, T), \chi(\mu)=x} I^{k_{t}}(\mu)=\inf _{s \in \mathbb{R}, \chi\left(\mu_{s}\right)=x} I^{k_{t}}\left(\mu_{s}\right)
$$




$$
=P(t)+\inf _{s \in \mathbb{R}, \chi\left(\mu_{s}\right)=x}\left\{t \chi\left(\mu_{s}\right)-h_{\mu_{s_{\chi}\left(\mu_{s}\right)}}(T)\right\},
$$

which gives (3.7). If $L^{*}$ has a subgradient at $x$, then it is unique (namely, the real $s$ such that $L^{\prime}(s)=x$ ), hence $L^{*}$ is strictly convex on the interior of its effective domain and essentially smooth ([20], Corollary 26.3.1). In all cases, the last assertion follows from Theorem $3.3 \mathrm{~b}$ ).

Remark 3.6. In the proof of Theorem [3.3, in order to get the upper bounds with rate function $Q_{f}{ }^{*}$, in view of Theorem 4.5.3 of [4] we only need the inequality $\overline{\mathbb{L}}^{*} \geq Q_{f}{ }^{*}$ in place of $(3.2)$, and therefore the inequality $\overline{\mathbb{L}}(\widehat{g}) \leq Q_{f}(g)$ for all $g$ in a dense subset of $C(J)$ is sufficient; in particular, the existence of $\mathbb{L}(g)$ is not necessary. Also, part b) of Theorem 3.3 follows from the upper bounds.

\section{EXAMPLES}

In this section we apply the preceding results to various sequences of measures on $\mathcal{M}(J)$ with powers $(1 / n)$. Our first example is analogue to those of [16] and [15] constructed by means of preimages and periodic points, respectively. The change consists in replacing these points by the elements of maximal separated sets, and allowing $f$ to be any element of $C(J)$; note that the techniques based on [10] do not work here when $f$ has more than one equilibrium state $(c f$. 1.3$)$. All the results obtained hold verbatim for preimages and periodic points, when $f$ is Hölder continuous ( $c f$. 4.2).

In order to handle the above atomic examples as well as the case of distribution of Birkhoff averages with respect to the measure of maximal entropy (Remark 4.1), we shall consider the following scheme. For each $y \in J$ and each integer $n \geq 1$ we define the measure $\mu_{y, n}=\frac{1}{n}\left(\delta_{y}+\ldots+\delta_{T^{n-1} y}\right)$, and for each $k \in C(J)$ we put $S_{n}(k)(y)=k(y)+\ldots+k\left(T^{n-1} y\right)$. We shall fix some $f \in C(J)$ and consider a suitable sequence $\left(J_{n}\right)$ of subspaces of $J$, each one provided with a Borel probability measure $p_{n, f}$, and set $\nu_{n, f}=W_{n}\left[p_{n, f}\right]$, where $W_{n}$ is the $\mathcal{M}(J)$-valued random variable on $J_{n}$ defined by $W_{n}(y)=\mu_{y, n}$. The large deviation principles are obtained from Theorem [3.3, once checked that $\left(\nu_{n, f}\right)$ fulfils the general hypothesis.

4.1. Separated sets, $f \in C(J)$. Let $f \in C(J)$, let $\varepsilon_{0}$ be the expansivity constant for $J$, let $\varepsilon<\varepsilon_{0} / 2$, let $J_{n}$ be a maximal $(\varepsilon, n)$-separated set, and let $p_{n, f}$ be the probability measure having mass $p_{n, f}(y)=\frac{e^{S_{n}(f)(y)}}{\sum_{z \in J_{n}} e^{S_{n}(f)(z)}}$ at each $y \in J_{n}$, so that $\nu_{n, f}=\sum_{y \in J_{n}} p_{n, f}(y) \delta_{\mu_{y, n}}\left(\right.$ note that $p_{n, f}=\operatorname{Card}\left(J_{n}\right)^{-1}$ when $\left.f=0\right)$.

4.1.1. Level-2 large deviation principles. Direct computations yield for each $g \in$ $C(J)$

$$
\frac{1}{n} \log \nu_{n, f}\left(e^{n \widehat{g}}\right)=\frac{1}{n} \log \sum_{y \in J_{n}} e^{S_{n}(f+g)(y)}-\frac{1}{n} \log \sum_{y \in J_{n}} e^{S_{n}(f)(y)},
$$

and since by definition of the pressure

$$
\lim \frac{1}{n} \log \sum_{y \in J_{n}} e^{S_{n}(k)(y)}=P(T, k)
$$

for all $k \in C(J)\left([22)\right.$, we get $\mathbb{L}(\widehat{g})=Q_{f}(g)$ by taking the limit in (4.1), and the hypothesis of Theorem 3.3 is fulfilled; consequently all the conclusions of this 
theorem hold. Explicitly, the large deviation principle means that

$$
\varlimsup \frac{1}{n} \log \sum_{y \in J_{n}, \mu_{y, n} \in F} p_{n, f}(y) \leq-\inf _{\mu \in F \cap \mathcal{M}(J, T)}\left\{P(T, f)-\mu(f)-h_{\mu}(T)\right\}
$$

for all closed sets $F \subset \mathcal{M}(J)$, and

$$
\varliminf \frac{1}{n} \log \sum_{y \in J_{n}, \mu_{y, n} \in G} p_{n, f}(y) \geq-\inf _{\mu \in G \cap \mathcal{M}(J, T)}\left\{P(T, f)-\mu(f)-h_{\mu}(T)\right\}
$$

for all open sets $G \subset \mathcal{M}(J)$. When $G \subset \mathcal{M}(J)$ is a convex open set containing some invariant measure, the lower limit in (4.4) is a limit and the inequality is an equality; furthermore, there exists a sequence of invariant measures $\left(\mu_{m}\right)$, each of which being the unique equilibrium state for some Hölder continuous function, converging weakly* and in entropy to some invariant measure $\mu \in \bar{G}$ realizing the infimum of $I^{f}$ on $\bar{G}$ and $G$, i.e. ,

$$
\begin{aligned}
& \lim \frac{1}{n} \log \sum_{y \in J_{n}, \mu_{y, n} \in G} p_{n, f}(y)=\lim \frac{1}{n} \log \sum_{y \in J_{n}, \mu_{y, n} \in \bar{G}} p_{n, f}(y) \\
& =\mu(f)+h_{\mu}(T)-P(T, f)=\lim \mu_{m}(f)+h_{\mu_{m}}(T)-P(T, f) .
\end{aligned}
$$

The R.H.S. of (4.3) is strictly negative when $F \cap \mathcal{M}_{f}(J, T)=\emptyset$. If furthermore we assume that $F$ is convex with nonempty interior, since $F$ is necessarily regular (24], pp. 38), we can apply (4.5) with $G=\operatorname{Int}(F)$ so that the upper limit in (4.3) is a limit and the inequality is an equality. The last assertion of Theorem 3.3 a) yields for each invariant measure $\mu$,

$$
h_{\mu}(T)=P(T, f)-\mu(f)+\lim _{\varepsilon \rightarrow 0} \lim \frac{1}{n} \log \sum_{y \in J_{n}, \rho\left(\mu, \mu_{y, n}\right)<\varepsilon} p_{n, f}(y),
$$

where $\rho$ is any distance on $\mathcal{M}(J)$ compatible with the weak ${ }^{*}$-topology, and for which the open balls are convex. Since

$$
\log \sum_{y \in J_{n}, \rho\left(\mu, \mu_{y, n}\right)<\varepsilon} p_{n, f}(y)=\log \sum_{y \in J_{n}, \rho\left(\mu, \mu_{y, n}\right)<\varepsilon} e^{S_{n}(f)(y)}-\log \sum_{z \in J_{n}} e^{S_{n}(f)(z)},
$$

and

$$
\lim \frac{1}{n} \log \sum_{z \in J_{n}} e^{S_{n}(f)(z)}=P(T, f)
$$

by (4.2), from (4.6) we get the following expression for the measure-theoretic entropy of $\mu$,

$$
h_{\mu}(T)=-\mu(f)+\lim _{\varepsilon \rightarrow 0} \lim \frac{1}{n} \log \sum_{y \in J_{n}, \rho\left(\mu, \mu_{y, n}\right)<\varepsilon} e^{S_{n}(f)(y)},
$$

and taking $f=0$,

$$
h_{\mu}(T)=\lim _{\varepsilon \rightarrow 0} \lim \frac{1}{n} \log \operatorname{Card}\left\{y \in J_{n}: \rho\left(\mu, \mu_{y, n}\right)<\varepsilon\right\} .
$$

When $f$ has a unique equilibrium state $\mu_{f}$ we obtain from Theorem $3.3 \mathrm{~b}$ ),

$$
\lim \sum_{y \in J_{n}} p_{n, f}(y) \mu_{y, n}=\mu_{f} .
$$


4.1.2. Level-1 large deviation principles. By applying Corollary 3.4 to the sequence $\left(\nu_{n, f}\right)$ and any $k \in C(J)$, we obtain the following large deviation results.

$$
\varlimsup \frac{1}{n} \log \sum_{y \in J_{n}, \frac{S_{n}(k)(y)}{n} \in C} p_{n, f}(y) \leq-\inf _{\mu \in \mathcal{M}(J, T), \mu(k) \in C}\left\{P(T, f)-\mu(f)-h_{\mu}(T)\right\}
$$

for all closed sets $C \subset \mathbb{R}$, and

$$
\underline{\lim } \frac{1}{n} \log \sum_{y \in J_{n}, \frac{S_{n}(k)(y)}{n} \in U} p_{n, f}(y) \geq-\inf _{\mu \in \mathcal{M}(J, T), \mu(k) \in U}\left\{P(T, f)-\mu(f)-h_{\mu}(T)\right\}
$$

for all open sets $U \subset \mathbb{R}$. For each $\mu \in \mathcal{M}(J, T)$ and each $\varepsilon$ small enough we have

$$
\begin{aligned}
& \lim \frac{1}{n} \log \sum_{y \in U_{n, \mu, \varepsilon}} p_{n, f}(y)=-\inf _{\mu^{\prime} \in G_{k, \mu, \varepsilon} \cap \mathcal{M}(J, T)}\left\{P(T, f)-\mu^{\prime}(f)-h_{\mu^{\prime}}(T)\right\} \\
& =\lim \frac{1}{n} \log \sum_{y \in \overline{U_{n, \mu, \varepsilon}}} p_{n, f}(y)=-\sum_{\mu^{\prime} \in \bar{G}_{k, \mu, \varepsilon} \cap \mathcal{M}(J, T)}\left\{P(T, f)-\mu^{\prime}(f)-h_{\mu^{\prime}}(T)\right\},
\end{aligned}
$$

with $U_{n, \mu, \varepsilon}=\left\{y \in J_{n}:\left|\frac{S_{n}(k)(y)}{n}-\mu(k)\right|>\varepsilon\right\}$ and $G_{k, \mu, \varepsilon}=\left\{\mu^{\prime} \in \mathcal{M}(J)\right.$ : $\left.\left|\mu^{\prime}(k)-\mu(k)\right|>\varepsilon\right\}$.

4.1.3. Lyapunov exponents. We assume here that $T$ is not conjugate to $z \mapsto z^{ \pm d}$, and we specialize 4.1 .2 by taking $f=k_{t}\left(t\right.$ any real) and $k=k_{-1}$, so that (4.10)(4.12) hold with $\log \left|T^{n^{\prime}}(y)\right|, \frac{\left|T^{n \prime}(y)\right|^{-t}}{\sum_{z \in J_{n}}\left|T^{\prime \prime}(z)\right|^{-t}}, \chi(\mu)$ in place of $S_{n}(k)(y), p_{n, f}(y)$, $\mu(k)$, respectively. Let us detail the case where $\mu=\mu_{t}$ in (4.12) (recall that $\mu_{t}$ is the unique equilibrium state for $k_{t}$ ), and first note that the members of (4.12) are strictly negative. Furthermore, (4.12) is specified with Theorem 3.5 since

$$
\sum_{y \in U_{n, \mu_{t}, \varepsilon}} p_{n, k_{t}}(y)=\nu_{n, k_{t}}\left(\left\{\mu \in \mathcal{M}(J):\left|\mu\left(k_{-1}\right)-\chi\left(\mu_{t}\right)\right|>\varepsilon\right\}\right)=\nu_{n, k_{t}}\left(G_{k_{-1}, \mu_{t}, \varepsilon}\right) .
$$

More precisely, from the properties of $I_{k_{-1}}^{k_{t}}$, and since

$$
P(t)=\lim \frac{1}{n} \log \sum_{z \in J_{n}}\left|T^{n^{\prime}}(z)\right|^{-t}
$$

by (4.2), we deduce the following relations for each $(t, s) \in \mathbb{R}^{2}$,

$$
h_{\mu_{s}}(T)-t \chi\left(\mu_{s}\right)= \begin{cases}\lim \frac{1}{n} \log \sum_{\left\{y \in J_{n}: \frac{\log \left|T^{n \prime}(y)\right|}{n}>\chi\left(\mu_{s}\right)\right\}}\left|T^{n^{\prime}}(y)\right|^{-t} & \text { if } s \leq t \\ \lim \frac{1}{n} \log \sum_{\left\{y \in J_{n}: \frac{\log \left|T^{n \prime}(y)\right|}{n}<\chi\left(\mu_{s}\right)\right\}} \mid T^{\left.n^{\prime}(y)\right|^{-t}} \quad \text { if } s \geq t,\end{cases}
$$

and for each real $t$ and each $\varepsilon>0$ small enough,

$$
\begin{aligned}
& \lim \frac{1}{n} \log \sum_{\left\{y \in J_{n}:\left|\frac{\log \mid T^{n^{\prime}(y) \mid}}{n}-\chi\left(\mu_{t}\right)\right|>\varepsilon\right\}} \frac{\left|T^{n^{\prime}}(y)\right|^{-t}}{\sum_{z \in J_{n}}\left|T^{n^{\prime}}(z)\right|^{-t}}= \\
& \max \left\{h_{\mu_{s_{\chi}\left(\mu_{t}\right)+\varepsilon}}(T)-t \varepsilon, h_{\mu_{s_{\chi}\left(\mu_{t}\right)-\varepsilon}}(T)+t \varepsilon\right\}-h_{\mu_{t}}(T) .
\end{aligned}
$$


Taking $s=t$ in (4.14) gives formulas for $P(t)$, and taking $t=0$ in (4.14) yields the following formula for the entropy valid for each real $s$,

$$
h_{\mu_{s}}(T)= \begin{cases}\lim \frac{1}{n} \log \operatorname{Card}\left\{y \in J_{n}: \frac{\log \left|T^{n \prime}(y)\right|}{n}>\chi\left(\mu_{s}\right)\right\} & \text { if } s \leq 0 \\ \lim \frac{1}{n} \log \operatorname{Card}\left\{y \in J_{n}: \frac{\log \left|T^{n \prime}(y)\right|}{n}<\chi\left(\mu_{s}\right)\right\} & \text { if } s \geq 0 .\end{cases}
$$

We obtain also from the last assertion of Theorem 3.5.

$$
\forall t \in \mathbb{R}, \quad \lim \sum_{y \in J_{n}} \frac{\left|T^{n^{\prime}}(y)\right|^{-t}}{\sum_{z \in J_{n}}\left|T^{n^{\prime}}(z)\right|^{-t}} \frac{\log \left|T^{n^{\prime}}(y)\right|}{n}=\chi\left(\mu_{t}\right) .
$$

Note that the strict inequality can be replaced by an inequality in (4.14), (4.15), (4.16); applying that to (4.14) with $s=t$, we recover (4.13).

4.2. Pre-images (resp. periodic points), $f$ Hölder continuous. Let $f$ be a Hölder continuous function on $J$, let $x \in J$, put $J_{n}=\left\{T^{-n}(x)\right\}$ (resp. $J_{n}=$ $\left\{y \in J: T^{n}(y)=y\right\}$ ), and let $\nu_{n, f}$ defined analogously to 4.1 namely $\nu_{n, f}=$ $\sum_{y \in J_{n}} p_{n, f}(y) \delta_{\mu_{y, n}}$ with $p_{n, f}(y)=\frac{e^{S_{n}(f)(y)}}{\sum_{z \in J_{n}} e^{S_{n}(f)(z)}}$ for all $y \in J_{n}$. Then (4.2) holds when $k$ is Hölder continuous ( $c f$. [18] for preimages, 14] for periodic points) and since $f+g$ is Hölder continuous when $g$ is, by taking the limit in (4.1) we get $\mathbb{L}(\widehat{g})=Q_{f}(g)$ for all such $g$, so that the hypothesis of Theorem 3.3 is fulfilled. Consequently, all the conclusions of 4.1.1 4.1 .2 and 4.1 .3 hold verbatim with $J_{n}$ as above. In particular, since $f$ has a unique equilibrium state $\mu_{f}$, (4.9) holds, and the members of (4.12) with $\mu=\mu_{f}$ are strictly negative.

Remark 4.1. The results of [11] concerning the Birkhoff averages with respect to the measure of maximal entropy $\mu_{0}$, can be easily recovered from Theorem 3.3 and Theorem 3.5. Indeed, take $f=0, J_{n}=J$ and $p_{n, 0}=\mu_{0}$ for all $n \geq 1$, so that

$$
\nu_{n, 0}(\cdot)=\mu_{0}\left(\left\{y \in J: \mu_{y, n} \in \cdot\right\}\right),
$$

and

$$
\lim \frac{1}{n} \log \nu_{n, 0}\left(e^{n \widehat{g}}\right)=\lim \frac{1}{n} \log \mu_{0}\left(e^{S_{n}(g)}\right)=Q_{0}(g)=P(g)-\log d
$$

for all $g \in C(J)$ ([11], Remark 1), hence the hypotheses of Theorem 3.3 is satisfied, and we recover the level-2 large deviation principle for $\left(\nu_{n, 0}\right)$ as in Theorem 7 of 11. In particular, the expression of the entropy is given for each $\mu \in \mathcal{M}(J, T)$ (and any distance $\rho$ as in (4.6) ) by

$$
h_{\mu}(T)=\log d+\lim _{\varepsilon \rightarrow 0} \lim \frac{1}{n} \log \mu_{0}\left(\left\{y \in J: \rho\left(\mu, \mu_{y, n}\right)<\varepsilon\right\}\right)
$$

(we note that in Remark 5 of [11] the term $\log d$ is missing). Assuming that $T$ is not conjugate to $z \mapsto z^{ \pm d}$, and taking $f=0$ and $k=k_{-1}$ in Theorem 3.5, the analogues of (4.14) and (4.15) are respectively for each real $s$,

$$
h_{\mu_{s}}(T)= \begin{cases}\log d+\lim \frac{1}{n} \log \mu_{0}\left(\left\{y \in J: \frac{\log \left|T^{n \prime}(y)\right|}{n}>\chi\left(\mu_{s}\right)\right\}\right) & \text { if } s \leq 0 \\ \log d+\lim \frac{1}{n} \log \mu_{0}\left(\left\{y \in J: \frac{\log \left|T^{n \prime}(y)\right|}{n}<\chi\left(\mu_{s}\right)\right\}\right) & \text { if } s \geq 0,\end{cases}
$$

and for each $\varepsilon>0$ small enough,

$$
\lim \frac{1}{n} \log \mu_{0}\left(\left\{y \in J:\left|\frac{\log \left|T^{n^{\prime}}(y)\right|}{n}-\chi\left(\mu_{0}\right)\right|>\varepsilon\right\}\right)=
$$




$$
\max \left\{h_{\mu_{s_{\chi\left(\mu_{0}\right)+\varepsilon}}}(T), h_{\mu_{s_{\chi}\left(\mu_{0}\right)-\varepsilon}}(T)\right\}-\log d .
$$

The formula (4.19) can easily be deduced from [11, and (4.20) corresponds to Corollary 2 of [11].

\section{Generalization - Examples}

It is easy to see that the proof of Theorem 3.3 does not depend on the dynamics of rational maps. In fact, it rests on two basic ingredients: the equality of functionals $\mathbb{L}(\cdot)=Q_{f}(\cdot)$, and the approximation property (given by Theorem 3.2) combined with Lemma 3.1. These conditions involving nets of measures on the phase space and notions of thermodynamical formalism, they can be defined (and Lemma 3.1 proved) as well for general dynamical systems in the sense of Ruelle's book (22]). In this section, after stating the general version (Theorem [5.2), we observe that the main result of [6] concerning the multidimensional full shift amounts to the approximation property, and thus furnishes an example of distinct nature from the one dimensional system given by rational maps, but sharing similar large deviation principles for the same kinds of measures with moreover the same proof (Theorem 5.7). Again here the techniques of [10] do not apply when $f$ has several equilibrium states.

Let $\Omega$ be a non-empty compact metrizable space, let $l$ be a strictly positive integer, put $\mathbb{Z}_{+}^{l}=\left\{x \in \mathbb{Z}^{l}: x_{i} \geq 0,1 \leq i \leq l\right\}$, and let $\tau$ be a representation of the semi-group $\mathbb{Z}_{+}^{l}$ (resp. group $\mathbb{Z}^{l}$ ) in the semi-group of continuous endomorphisms (resp. group of homeomorphisms) of $\Omega$. Let $C(\Omega), \mathcal{M}(\Omega), \mathcal{M}^{\tau}(\Omega), \mathcal{M}_{f}^{\tau}(\Omega), h^{\tau}$, $P^{\tau}(\cdot)$ be the obvious analogues of $C(J), \mathcal{M}(J), \mathcal{M}(J, T), \mathcal{M}_{f}(J, T), h .(T), P(T, \cdot)$ defined in 93 , and assume that $h^{\tau}$ is finite and upper semi-continuous. For each $f \in C(\Omega)$ we define the function $I^{f}$ on $\mathcal{M}(\Omega)$ by

$$
I^{f}(\mu)= \begin{cases}P^{\tau}(f)-\mu(f)-h^{\tau}(\mu) & \text { if } \mu \in \mathcal{M}^{\tau}(\Omega) \\ +\infty & \text { if } \mu \in \mathcal{M}(\Omega) \backslash \mathcal{M}^{\tau}(\Omega),\end{cases}
$$

so that $I^{f}$ vanishes exactly on $\mathcal{M}_{f}^{\tau}(\Omega)$. The analogue of the approximation property of Theorem 3.2 takes the following general form.

Property 5.1. For each $\mu \in \mathcal{M}^{\tau}(\Omega)$ there is a net $\left(k_{i}\right)_{i \in \wp_{\mu}}$ in $C(\Omega)$ such that $k_{i}$ has a unique equilibrium state $\mu_{i}$ for all $i \in \wp_{\mu}$, and the net $\left(\mu_{i}\right)_{i \in \wp_{\mu}}$ satisfies $\lim \mu_{i}=\mu$ and $\lim h_{\mu_{i}}^{\tau}=h_{\mu}^{\tau}$.

We can now state the general version of Theorem 3.3. whose proof is entirely similar (just take account of Remark 3.6 for a), and use Property 5.1 in place of Theorem 3.2 for b)). We let the reader establish the analogue of Corollary 3.4 .

Theorem 5.2. Let $f \in C(\Omega)$, let $\left(\nu_{\alpha}\right)$ be a net of Borel probability measures on $\mathcal{M}(\Omega)$, let $\left(t_{\alpha}\right)$ be a net in $] 0,+\infty[$ converging to 0 , let $\overline{\mathbb{L}}$ be the associated large deviation functional, and assume there is a dense set $C \subset C(\Omega)$ such that

$$
\forall g \in C, \quad \overline{\mathbb{L}}(\widehat{g}) \leq P^{\tau}(f+g)-P^{\tau}(f) .
$$

a) For each closed set $F \subset \mathcal{M}(\Omega)$ we have

$$
\varlimsup t_{\alpha} \log \nu_{\alpha}(F) \leq-\inf _{\mu \in F} I^{f}(\mu) .
$$


Each limit point of $\left(\nu_{\alpha}\right)$ has its support included in $\mathcal{M}_{f}^{\tau}(\Omega)$; in particular, $\lim \nu_{\alpha}=\delta_{\mu_{f}}$ when $f$ has a unique equilibrium sate $\mu_{f}$.

b) If Property 5.1 holds and $\mathbb{L}(\widehat{g})$ exists for all $g \in C$ with an equality in (5.1), then for each open set $G \subset \mathcal{M}(\Omega)$ we have

$$
\underline{\lim } t_{\alpha} \log \nu_{\alpha}(G) \geq-\inf _{\mu \in G} I^{f}(\mu)
$$

when $G$ is moreover convex and contains some invariant measure, we have

$$
\lim t_{\alpha} \log \nu_{\alpha}(G)=\lim t_{\alpha} \log \nu_{\alpha}(\bar{G})=-\inf _{\mu \in \bar{G}} I^{f}(\mu)=-\inf _{\mu \in G \cap \mathcal{E}^{\prime}} I^{f}(\mu),
$$

where $\mathcal{E}^{\prime}$ is the set of equilibrium states of all elements in $\left\{k_{i}: i \in \wp_{\mu}, \mu \in\right.$ $\left.\mathcal{M}^{\tau}(\Omega)\right\}$. In particular we obtain for each $\mu \in \mathcal{M}^{\tau}(\Omega)$ and each convex local basis $\mathcal{G}_{\mu}$ at $\mu$,

$$
h^{\tau}(\mu)=P^{\tau}(f)-\mu(f)+\inf \left\{\lim t_{\alpha} \log \nu_{\alpha}(G): G \in \mathcal{G}_{\mu}\right\} .
$$

Remark 5.3. Property 5.1 permits to get more than just a large deviation principle with rate function $I^{f}$. Indeed, although the two first equalities in (5.2) are still true without Property 5.1 (combining (2.1) with the convexity of $G$ and the fact that $I^{f}$ is affine), the last equality is given by Property [5.1, and shows that the exponential behavior of $\nu_{\alpha}(G)$ is controlled by the entropy of measures which are unique equilibrium states. More precisely, for each $\varepsilon>0$ there exists $k_{\varepsilon} \in C(\Omega)$ with unique equilibrium state $\mu_{k_{\varepsilon}} \in G$ such that eventually,

$$
\left|t_{\alpha} \log \nu_{\alpha}(G)+I^{f}\left(\mu_{k_{\varepsilon}}\right)\right|<\varepsilon .
$$

Remark 5.4. Condition (5.1) corresponds to Assumptions (a), (b) of [17] for suitable measures and $f=0$, and consequently Theorem 2 (resp. Corollary 2.1) of [17] follows immediately from the first (resp. second) assertion of Theorem 5.2 a).

Remark 5.5. Theorem 5.2 a) can be applied to the same dynamics as the one considered in 33 with $T$ any rational map of degree $d \geq 2$ (without hyperbolicity condition). For instance, let us consider the sequence of measures obtained with preimages and $f$ Hölder continuous as in 4.2. It is known that (4.2) still holds when $k$ is Hölder continuous and $P(T, k)>\sup _{J} k$, and for general $k \in C(J)$ the equality in (4.2) has to be replaced by " $\leq$ " and the limit by a upper limit (18]). Consequently, when $P(T, f)>\sup _{J} f$, (5.1) is fulfilled with $C$ the set of Hölder continuous functions by taking the upper limit in (4.1). As a conclusion, we recover the large deviation results of [16].

5.1. The multidimensional full shift. Let $S$ be a finite set, let $\delta \in] 0,1[$, put $\Omega=S^{\mathbb{Z}^{l}}$ endowed with the metric $\rho(\xi, \eta)=\delta^{\min \left\{\max _{1 \leq i \leq l}\left|x_{i}\right|: x \in \mathbb{Z}^{l}, \xi_{x} \neq \eta_{x}\right\}}$, and let $\tau$ be the action of $\mathbb{Z}^{l}$ on $\Omega$ by translations, i.e. $\left(\tau^{y} \xi\right)_{x}=\xi_{x+y}$ for all $x, y$ in $\mathbb{Z}^{l}$ and $\xi \in \Omega$. A recent result of Gurevich and Tempelman ([6], Theorem 1) can be formulated in the following way (in fact, the authors show that the continuous functions appearing in Property 5.1 can be obtained as mean energy functions associated to some summable interactions).

Theorem 5.6. (Gurevich-Tempelman) The multidimensional full shift fulfils Property 5.1 . 
We shall consider nets of measures similar to those of 4.1 .1 and 4.2 The "time" $n$ is replaced by a multidimensional one, namely a net $\left(\Lambda_{\alpha}\right)_{\alpha \in \wp}$ of finite subsets of $\mathbb{Z}^{l}$. Recall that $\left(\Lambda_{\alpha}\right)$ is said to converge to $\infty$ in the sense of van Hove (denoted $\Lambda_{\alpha} \nearrow$ $\infty)$ when $\lim \left|\Lambda_{\alpha}\right|=+\infty$ (where $\left.\left|\Lambda_{\alpha}\right|=\operatorname{Card} \Lambda_{\alpha}\right)$ and $\lim \left|\left(\Lambda_{\alpha}+x\right) \backslash \Lambda_{\alpha}\right| /\left|\Lambda_{\alpha}\right|=0$ for all $x \in \mathbb{Z}^{l}$. Put $\mathbb{Z}_{>}^{l}=\left\{x \in \mathbb{Z}^{l}: x_{i}>0,1 \leq i \leq l\right\}$, and for each $x \in \mathbb{Z}_{>}^{l}$ define $\Lambda(x)=\left\{y \in \mathbb{Z}^{l}: 0 \leq y_{i}<x_{i}, 1 \leq i \leq l\right\}$ and the set of $x$-periodic configurations $\operatorname{Per}_{x}=\left\{\xi \in \Omega: \tau^{y} \xi=\xi\right.$ for all $\left.y \in \mathbb{Z}^{l}(x)\right\}$, where $\mathbb{Z}^{l}(x)$ is the subgroup of $\mathbb{Z}^{l}$ generated by $\left(x_{1}, 0, \ldots, 0\right), \ldots,\left(0, \ldots, 0, x_{l}\right)$. Note that $\Lambda(x) \nearrow \infty$ when $\mathbb{Z}_{>}^{l}$ is directed by the lexicographic order and $\lim x_{i}=+\infty$ for all $i \in\{1, \ldots, l\}$, which will be assumed in what follows. For each $\xi \in \Omega$ we define

$$
\mu_{\xi, \alpha}=\frac{1}{\left|\Lambda_{\alpha}\right|} \sum_{x \in \Lambda_{\alpha}} \delta_{\tau^{x} \xi}
$$

and

$$
W_{\alpha}(\xi)=\mu_{\xi, \alpha} .
$$

In place of the sequences $\left(J_{n}\right)$ we shall consider some nets $\left(\Omega_{\alpha}\right)$ of finite subsets of $\Omega ; \mu_{y, n}$ (resp. $\left.W_{n}\right)$ is replaced by $\mu_{\xi, \alpha}$ (resp. $W_{\alpha}$ ), and the probability measures $p_{n, f}$ by $p_{\alpha, f}$ with

$$
p_{\alpha, f}(\xi)=\frac{e^{\sum_{x \in \Lambda_{\alpha}} f\left(\tau^{x} \xi\right)}}{\sum_{\xi^{\prime} \in \Omega_{\alpha}} e^{\sum_{x \in \Lambda_{\alpha}} f\left(\tau^{x} \xi^{\prime}\right)}} .
$$

We shall obtain large deviations for nets $\left(\nu_{\alpha, f}\right)$ defined by

$$
\nu_{\alpha, f}=W_{\alpha}\left[p_{\alpha, f}\right]=\sum_{\xi \in \Omega_{\alpha}} p_{\alpha, f}(\xi) \delta_{\mu_{\xi, \alpha}},
$$

each of which is associated with some net $\Lambda_{\alpha} \nearrow \infty$ and corresponds to some way to obtain the pressure, in the sense that

$$
\forall g \in C(\Omega), \quad \mathbb{L}(\widehat{g})=\lim \frac{1}{\left|\Lambda_{\alpha}\right|} \log \nu_{\alpha, f}\left(e^{\left|\Lambda_{\alpha}\right| \widehat{g}}\right)=P^{\tau}(f+g)-P^{\tau}(f) .
$$

Once proved the above equality, the conclusion follows from Theorem 5.2 and Theorem 5.6 .

The next result establishes the large deviation principle for finite supported measures constructed from maximal separated sets (resp. periodic configurations) as for rational maps. The explicit forms of the large deviations as well as the formulas for entropy are entirely analogue to those for rational maps, after obvious changes of notations ( $c f$. 4.1.1 4.2 ). Both cases are new, and the one of periodic configurations generalizes the version of [5] and [13] proved for $f=0$ ( $c f$. Remark 5.8).

Theorem 5.7. Let $\Lambda_{\alpha} \nearrow \infty$ and $\left(\Omega_{\alpha}\right)$ given in one of the following ways.

(a) $\Omega_{\alpha}$ is a maximal $\left(\varepsilon, \Lambda_{\alpha}\right)$-separated set for some $\varepsilon<\delta$;

(b) $\Lambda_{\alpha}=\Lambda(\alpha)$ and $\Omega_{\alpha}=\operatorname{Per}_{\alpha}$ for all $\alpha \in \mathbb{Z}_{>}^{l}$.

Then for each $f \in C(\Omega)$, all the conclusions of Theorem 5.2 hold with $t_{\alpha}=\left|\Lambda_{\alpha}\right|^{-1}$ and

$$
\nu_{\alpha, f}=\sum_{\xi \in \Omega_{\alpha}} p_{\alpha, f}(\xi) \delta_{\mu_{\xi, \alpha}}
$$


Proof. Since

$$
\forall g \in C(\Omega), \quad \nu_{\alpha, f}\left(e^{\left|\Lambda_{\alpha}\right| \widehat{g}}\right)=\frac{\sum_{\xi \in \Omega_{\alpha}} e^{\sum_{x \in \Lambda_{\alpha}}(f+g)\left(\tau^{x} \xi\right)}}{\sum_{\xi^{\prime} \in \Omega_{\alpha}} e^{\sum_{x \in \Lambda_{\alpha}} f\left(\tau^{x} \xi^{\prime}\right)}},
$$

in both cases (5.3) follows from Theorem 2.2 of [23].

Remark 5.8. The results of Theorem 5.7 (b) are similar to those of [13], where nets of the form $\left(W_{\alpha}\left[\mu^{\mathbb{Z}^{l}}\right]\right)$ are studied, for some fixed probability measure $\mu$ on $S$. More precisely, when the spin space in [13 is finite and $\mu$ is the uniform distribution, Theorem 3.5 (resp. Theorem 4.2) of that paper is exactly the large deviation upper (resp. lower) bounds of Theorem 5.7 (b) with $f=0$. Therefore, Theorem 5.7 (b) generalizes this particular case allowing any $f \in C(\Omega)$; it also extends in the same way the full shift case in Theorem $\mathrm{C}$ of [5] where only $f=0$ is considered (on the other hand, Theorem $\mathrm{C}$ of [5] is much more general since it holds for any subshift of finite type satisfying strong specification).

Remark 5.9. The measures $p_{\alpha, f}$ as in Theorem [5.7 (b) have been considered in 23 for any $f \in C(\Omega)$; in particular, Theorem 3.2 of that paper establishes that every limit point of $\left(p_{\alpha, f}\right)$ belongs to $\mathcal{M}_{f}^{\tau}(\Omega)$. It is easy to see that this result can be recovered from Theorem 5.7. Indeed, let $\mu$ be a limit point of $\left(p_{\alpha, f}\right)$, or equivalently a limit point of $\left(\sum_{\xi \in \operatorname{Per}_{\alpha}} p_{\alpha, f}(\xi) \mu_{\xi, \alpha}\right)$. Then $\mu$ is the barycenter of some limit point $\nu$ of $\left(\nu_{\alpha, f}\right)$ (recall that the barycenter map is weak* continuous). Since $\nu$ is supported by $\mathcal{M}_{f}^{\tau}(\Omega)$ (Theorem $[5.2 \mathrm{a}$ )), we conclude that $\mu$ belongs to $\mathcal{M}_{f}^{\tau}(\Omega)$.

Acknowledgments. The author wishes to thank Juan Rivera-Letelier for many helpful discussions on the dynamics of rational maps. Thanks are also due for the financial support and the warm hospitality during various visits at the Universidad Católica del Norte. This work has been supported by FONDECYT grant No. 1070045 .

\section{REFERENCES}

[1] P. Baldi. Large deviations and stochastic homogenization. Ann. Mat. Pura Appl. 151 (1988), 161-17\%.

[2] H. Comman. Variational form of the large deviation functional. Statistics and Probability Letters 77 (2007), no. 9 , 931-936.

[3] H. Comman. Criteria for large deviations. Trans. Amer. Math. Soc. 355 (2003), no. 7, 2905-2923.

[4] A. Dembo, O. Zeitouni. Large deviations techniques and applications, Second Edition, Springer, New-York, 1998.

[5] A. Eizenberg, Y. Kifer, B. Weiss. Large deviations for $\mathbb{Z}^{d}$-actions. Comm. Math. Phys 164 (1994), 433-454.

[6] B. M. Gurevich, A. A. Tempelman. Markov approximation of homogeneous lattice random fields. Probab. Theory Related Fields 131 (2005), 519-52\%.

[7] G. Keller. Equilibrium states in ergodic theory, London Mathematical Society Student Texts 42, Cambridge University Press, Cambridge, 1998.

[8] G. Keller, T. Nowicki. Spectral theory, Zeta functions and the distribution of periodic points for Collet-Eckmann maps. Comm. Math. Phys. 149 (1992), 31-69.

[9] Y. Kifer. Large deviations, averaging and periodic orbits of dynamical systems. Comm. Math. Phys. 162 (1994), no. 1, 33-46.

[10] Y. Kifer. Large deviations in dynamical systems and stochastic processes. Trans. Amer. Math. Soc. 321 (1990), no. 2, 505-524. 
[11] A. O. Lopes. Entropy and large deviation. Nonlinearity 3 (1990), 527-546.

[12] I. Melbourne, M. Nicol. Large deviations for nonuniformly hyperbolic systems. Trans. Amer. Math. Soc. 360 (2008), 6661-6676.

[13] S. Olla. Large deviations for Gibbs random fields. Probab. Theory and Related Fields 77 (1988), 343-35\%.

[14] W. Parry, M. Pollicott. Zeta functions and the periodic orbit structure of hyperbolic dynamics, Asterisque 187-188 (1990) ISSN: 0303-1179.

[15] M. Pollicott. Large deviations results for periodic points of a rational map. J. Dyn Syst. Geom. Theor. 5 (2007), no. 1, 69-67.

[16] M. Pollicott, R. Sharp. Large deviations and the distribution of preimages of rational maps. Comm. Math. Phys. 181 (1996), 733-739.

[17] M. Pollicott. Closed geodesic distribution for manifolds of non-positive curvature, Discrete and Continuous Dynamical Systems 2 (1996), no. 2, 153-161.

[18] F. Przytycki. On the Perron-Frobenius-Ruelle operator for rational maps on the Riemann sphere and for Hölder continuous functions. Bol. Bras. Mat. Soc. 20 (1990), 95-125.

[19] L. Rey-Bellet, L. S. Young. Large deviations in non-uniformly hyperbolic dynamical systems. Ergod. Th. Dynam. Sys. 28 (2008), 587-612.

[20] R. T. Rockafeller. Convex analysis, Princeton University Press, Princeton, 1970.

[21] D. Ruelle. Repellers for analytic maps. Ergod. Th. Dynam. Sys. 2 (1982), $9910 \%$.

[22] D. Ruelle. Thermodynamic formalism, Addison-Wesley, Reading, MA, 1978.

[23] D. Ruelle. Statistical mechanics on a compact set with $\mathbb{Z}^{d}$-action satisfying expansiveness and specification. Trans. Amer. Math. Soc. 185 (1973), 237-251.

[24] H. H. Schaefer. Topological vector spaces, Springer-Verlag, New York, 1964.

Department of Mathematics, University of Santiago de Chile, Bernardo O'Higgins 3363, Santiago, Chile

E-mail address: hcomman@mat.usach.cl 